\title{
Equipamentos para Estimulação Elétrica Funcional
}

\author{
Antônio Cardoso Santos * \\ Danton P. Silva Jr. ** \\ André Frota Müller *** \\ Paulo R.Oppermann Thomé **** \\ Paulo Roberto Stefani Sanches ***** \\ Maria E. Alves $* * * * * *$ \\ Maria E. Bortolozzo $* * * * * * *$
}

\section{RESUMO}

Título: Estimulação Elétrica Funcional (FES)

Objetivos: Avaliação da técnica FES em pacientes com patologias neurológicas diversas, utilizando equipamentos desenvolvidos pela Engenharia Biomédica do Hospital de Clínicas de Porto Alegre. Os objetivos principais deste trabalho são: redução da espasticidade muscular, fortalecimento muscular e melhora no padrão de marcha.

Material: FES-II portátil, 2 canais, Engenharia Biomédica - HCPA

FES-II clínico, 2 canais, Engenharia Biomédica - HCPA

Estes equipamentos FES possuem as seguintes características principais:

Canais:

Amplitude da corrente de saída:

Largura dos pulsos:

Freqüência dos pulsos:

Trem de pulsos (Tempos de ataque, sustentação,

descida e repouso):

Controle Automático e Manual

Marcha automaticamente assistida (utilizando duas palmilhas especialmente desenvolvidas).

Métodos: Metodologia da Escola de Lubljana. Sessões periódicas de 10 minutos cada, aplicadas a pacientes previamente selecionados. Os resultados foram avaliados pela análise dos padrões de marcha e o exame clínico dos músculos estimulados.

\footnotetext{
* Médico Fisiatra HCPA. Professor Assistente em Reabilitação Médica do Departamento de Cirurgia FAMED/UFRGS. Chefe do Serviço de Fisiatria HCPA.

** Engenheiro Eletro-Eletrônico UFRGS/HCPA. Mestrando do Curso de PósGraduação em Matemática UFRGS.

*** Técnico HCPA. Acadêmico da FEE da PUC.

**** Engenheiro Eletro-Eletrônico UFRGS/HCPA. Pós-Graduação em Sistemas Digitais e Ciência da Computação UFRGS.

***** Engenheiro Eletro-Eletrônico UFRGS/HCPA. Especialização em Engenharia da Qualidade. Chefe do Setor de Engenharia Biomédica HCPA.

****** Fisioterapeuta HCPA.

******* Médico Fisiatra HCPA. Especialização em Medicina do Trabalho UFRGS Hospital de Clínicas de Porto Alegre (HCPA)
} 


\section{Resultados:}

Redução da espasticidade muscular:

$81,8 \%$ pacientes

Melhora no padrão de marcha:

$14 / 19$

$73,7 \%$ pacientes

Alívio no ombro doloroso:

$12 / 12$

$100 \%$ pacientes*

Conclusões: A técnica de Estimulação Elétrica Funcional (FES) é um meio eficiente de obter contrações musculares controladas em membros paralisados, e, se possível, restaurar as funções destes membros. FES é clinicamente indicado para pacientes com lesões na medula espinhal (04), acidentes vasculares cerebrais, paralisia central, esclerose múltipla, etc...(05). A aplicação regular da estimulação neuromuscular, usando FES, provou ser eficiente no tratamento de pacientes com deficiências motoras causadas por diferentes patologias do sistema nervoso central.

\section{UNITERMOS}

FES. Estimulação Elétrica Funcional. Eletroestimulação. Reabilitação Física. Redução da Espasticidade. Melhora no Padrão de Marcha.

\section{SUMMARY}

Title: Functional Electrical Stimulation (FES)

Aims: Evaluation of FES technique in patients with different neurologic pathologies, using devices developed by the Biomedical Engineering Division HCPA. The basic aims are: muscular spasticity reduction, muscle strengthening and gait pattern improvement.

Material: FES-II portable, 2 channels, Biomedical Engineering Division - HCPA

\section{Introdução}

A utilização de correntes elétricas na Medicina é antiga e bastante difundida. Desde que se descobriu que os tecidos biológicos excitáveis eram ativados por diferenças de potencial, vemse estudando maneiras de medir estes potenciais e reproduzi-los. No campo da Fisiatria, as correntes galvânica e farádica existem desde os primórdios da especialidade.

No início, a estimulação elétrica funcional foi usada com o objetivo de reduzir a espasticidade (e outros sintomas de fenômenos de liberação) através da excitação de músculos isolados. Atualmente, a estimulação elétrica funcional representa um método clinicamente eficaz na reabilitação de pacientes com lesão do neurônio motor superior.

Internacionalmente, a Estimulação Elétrica Funcional vem sendoestudada e utilizada com êxito desde 1961, obtendo melhores respostas em
FES-II clinical, 2 channels, Biomedical Engineering Division - HCPA

These FES equipments have the following characteristics:

Channels: $\quad 2$ isolated channels

Current output amplitude: 0 to $80 \mathrm{~mA}$

Pulse width: $\quad 100$ to 700

Pulse frequency: $\quad 10$ to $60 \mathrm{~Hz}$

Burst controls: Atack, sustain, decay and resting times: adjustables

Automatic and manual control

Automatically assisted human gait using two specially designed foot-palms.

Mehtods: Lubljana method. Periodic sessions of 10 minutes each applied to selected patients. The results were evaluated according to gait pattern analysis and clinical examinations of the stimulated muscles.

Results:

Muscular spasticity reduction: $\quad 27 / 33 \quad 81,8 \%$ patients

Gait pattern improvement: $14 / 1973,7 \%$ patients Shoulder pain relief: $\quad 12 / 12100 \%$ patients Conclusions: Functional Electrical Stimulation (FES) technique is an effective means of obtaining muscular contractions of the paralyzed limbs, and, if possible, restoring controllable limb function in high-level spinal cord injury patients. FES is clinically indicated for patients with: spinal cord injury ${ }^{4}$, cerebrovascular disorders (stroke), central paralysis, brain injury, multiple sclerosis, etc... ${ }^{5}$.

\section{KEYWORDS}

FES. Functional Electrical Stimulations. Electrostimulation. Physical Rehabilitations. Spasticity Reduction. Gait Pattern Improvement.

pacientes hemiplégicos, portadores de esclerose múltipla, paralisia cerebral e lesão medular.

No Brasil, a estimulação elétrica funcional foi introduzida em 1989 pelo Prof. Sérgio Lianza da Santa Casa de São Paulo ${ }^{13}$, seguindo a técnica da Universidade de Lubljana - Iugoslávia (Dr. Franco Gracanin e Dr. Tajed Badj), que forneceu suporte tecnológico para o desenvolvimento de um equipamento de fabricação nacional.

No Hospital de Clínicas de Porto Alegre (HCPA) a técnica de estimulação elétrica funcional foi introduzida em 1991.

Esta técnica é um dos avanços da Bioengenharia que mais modificou o perfil da reabilitação, por seu espectro de aplicação. É evidente que o FES é mais freqüentemente usado como uma ajuda ortótica com efeitos terapêuticos, fazendo, dentro do possível, o controle das contrações dos músculos e movimentos ${ }^{01,02}$, e, ao mesmo tempo, contribuindo para a organização dos mecanismos básicos que regulam os reflexos motores ${ }^{03}$. A sua 
aplicação na Medicina, entretanto, não se restringe ao sistema músculo-esquelético, sendo também utilizada como marcapasso cardíaco, marcapasso diafragmático (respiração), vesical (controle urinário), intestinal e até sexual. Em alguns casos, esta técnica poderá ser usada através de eletrodos implantados.

Com a evolução da tecnologia e o aprimoramento dos estudos neurofisiológicos, os novos equipamentos que surgem oferecem um enorme (e crescente) campo de pesquisa, diagnóstico e tratamento, além daqueles que unem a captação do estímulo com a sua potencialização (biofeedback).

Para utilização da metodologia FES são necessários: músculos e neurônio motor inferior íntegros. Estas condições primordiais só podem ser avaliadas no exame físico minuscioso e, em alguns casos, depender de exames complementares. Além dos fatores referentes ao próprio aparelho locomotor, é necessário que o paciente tenha condições de compreensão e participação adequados para entender o que se pretende com o tratamento, visto que este é um processo de reaprendizagem.

A técnica FES diferencia-se da eletroterapia convencional, basicamente, por possuir um perfil de corrente elétrica bipolar capaz de excitar a fibra nervosa tanto aferente (que vai para o Sistema Nervoso Central), quanto eferente (que vai para o músculo), e ser utilizada com o intuito de produzir contração muscular próxima à fisiológica e funcionalmente útil ${ }^{01,06}$.

O perfil da corrente FES faz com que o estímulo chegue ao Sistema Nervoso Central e a partir daí produza movimento ativo no paciente. Portanto, este movimento, além de produzir melhora do trofismo muscular, é capaz de servir para reaprendizado, uma vez que todo o esquema de movimento é percebido como sendo próprio. As técnicas neurofisiológicas aplicadas em cinesioterapia buscam exatamente este reaprendizado para ocupação das áreas vazias do cérebro (Diásquises).

O tipo de contração obtido pelo FES quando usado sobre o nervo eferente (que vai direto ao músculo) faz com que a técnica possa ser utilizada de modo contínuo, agindo com órtese. Estas órteses têm a vantagem de ser ativas, isto é, a própria musculatura do paciente coloca o segmento corporal afetado na posição desejada, funcionando também como tratamento. Assim, o uso de FES pode determinar aquisições permanentes, tanto de massa muscular, quanto de melhora no padrão motor do paciente.

\section{Objetivos}

Este trabalho tem como objetivos:

- Verificar a evolução dos pacientes em tratamento no Centro de Reabilitação do Hospital de Clínicas de Porto Alegre, utilizando equipamentos FES especialmente projetados e desenvolvidos pela Engenharia Biomédica do próprio HCPA.

- Validar os equipamentos FES de dois canais (modelo clínico e modelo portátil), desenvolvidos pela Engenharia Biomédica do HCPA, cujas especificações técnico-operacionais foram definidas em conjunto com o Setor de Fisiatria, de acordo com os mais recentes artigos científicos e prática terapêtica, incorporando ainda modernos dispositivos de segurança para operadores e pacientes. Os equipamentos nacionais portáteis, até então disponíveis no mercado, eram alimentados pela rede elétrica e não possuíam disparadores manuais ou de palmilha, que são acessórios imprescindíveis para a marcha recíproca e o uso do FES como órtese ${ }^{07,08}$.

- Permitir o desenvolvimento de pesquisas e trabalhos científicos relacionados à área de Estimulação Elétrica Funcional.

- Alguns objetivos da aplicação regular de estimuladores funcionais FES: Melhora da amplitude articular; Melhora da trofia muscular e força ${ }^{09}$; Aumento dos movimentos voluntários; Redução da espasticidade ${ }^{10,11}$; Retardo ou prevenção de contraturas e deformidades; Melhora do padrão de marcha ${ }^{07,12}$; Melhora do equilíbrio de tronco; Melhora da circulação local; Recurso ortésico.

\section{Materiais e Métodos}

A realização deste trabalho conjunto entre os setores de Engenharia Biomédica e Fisiatria do HCPA exigiu a definição de uma metodologia que envolvesse mais do que o simplès registro dos resultados médicos obtidos em pacientes com indicação terapêutica para o uso de estimuladores FES. Era necessária uma avaliação do desempenho, praticidade e eficiência dos equipamentos, visando a sua completa validação como um instrumento FES adequado às exigências encontradas no serviço de Fisiatria do hospital.

Este trabalho foi dirigido para pacientes portadores de seqüelas de patologias neurológicas, decorrentes de lesão do neurônio motor superior, que estavam em tratamento no Centro de Reabilitação do Hospital de Clínicas de Porto Alegre. A técnica de Cinesioterapia foi utilizada conjuntamente.

Os equipamentos FES desenvolvidos pela Engenharia Biomédica do HCPA possuem as seguintes características técnico-operacionais:
Número de Canais: 
Corrente de saída: $\quad 0$ a $80 \mathrm{~mA}$

Largura dos Pulsos: $\quad 100$ a $700 \mu \mathrm{s}$

Freqüência dos Pulsos: $10 \mathrm{a} 60 \mathrm{~Hz}$

Envelope FES (Ataque, sustentação, descida e repouso): ajustáveis

Modos de Operação: Automático e Manual

Eletrodos transcutâneos de borracha de silicone condutora

Conector para palmilhas especiais com sensores (marcha automaticamente assistida).

Os estimuladores FES desenvolvidos pela Engenharia Biomédica do HCPA foram utilizados durante sessões periódicas de 10 minutos, 3 vezes por semana, em um grupo de pacientes selecionados.

No período compreendido entre fevereiro de 1992 e maio de 1995, entraram em tratamento 58 pacientes com lesão neurológica, divididos de acordo com as seguintes patologias:

- 38 pacientes com acidente vascular-cerebral: $65,51 \%$

- 15 pacientes com traumatismo raquimedular: $25,86 \%$

- 2 pacientes com traumatismo crânio-encefálico: $3,45 \%$

- 2 pacientes com paralisia celebral: $3,45 \%$

- 1 paciente com esclerose múltipla: 1,72\%

TOTAL: 58 pacientes $\quad 100 \%$

Através de uma avaliação clínica, realizada pelo fisiatra, foram considerados fatores de inclusão para a amostra, entre eles: o tempo e o nível de lesão neurológica, o grau de espasticidade, a amplitude articular, e as condições clínicas favoráveis. Foi utilizada a metodologia da Escola de Lubljana.

A estimulação elétrica funcional foi realizada durante 10 minutos, no local determinado, associada à cinesioterapia. As revisões foram realizadas a cada vinte sessões.

\section{Pacientes hemiplégicos:}

12 apresentavam quadro de ombro doloroso associado ao eqüinismo, portanto foram estimulados os pontos motores do deltóide e dorsifexores do pé;

14 apresentavam espasticidade flexorà em membro superior associada ao eqüinismo, portanto foram estimulados os pontos motores do tríceps braquial e extensores de punho e dedos e dorsiflexores do pé;

12 apresentavam ombro doloroso sendo estimulados os pontos motores do deltóide.

\section{Pacientes com lesão medular:}

15 foram estimulados bilateralmente no quadríceps;

02 foram estimulados os pontos motores no quadríceps e glúteos máximos.

\section{Sinal FES na saída dos equipamentos}

\section{Largura de Pulso de 0,1 a 0,7 ms}

Freqüência entre 10 e $60 \mathrm{~Hz}$
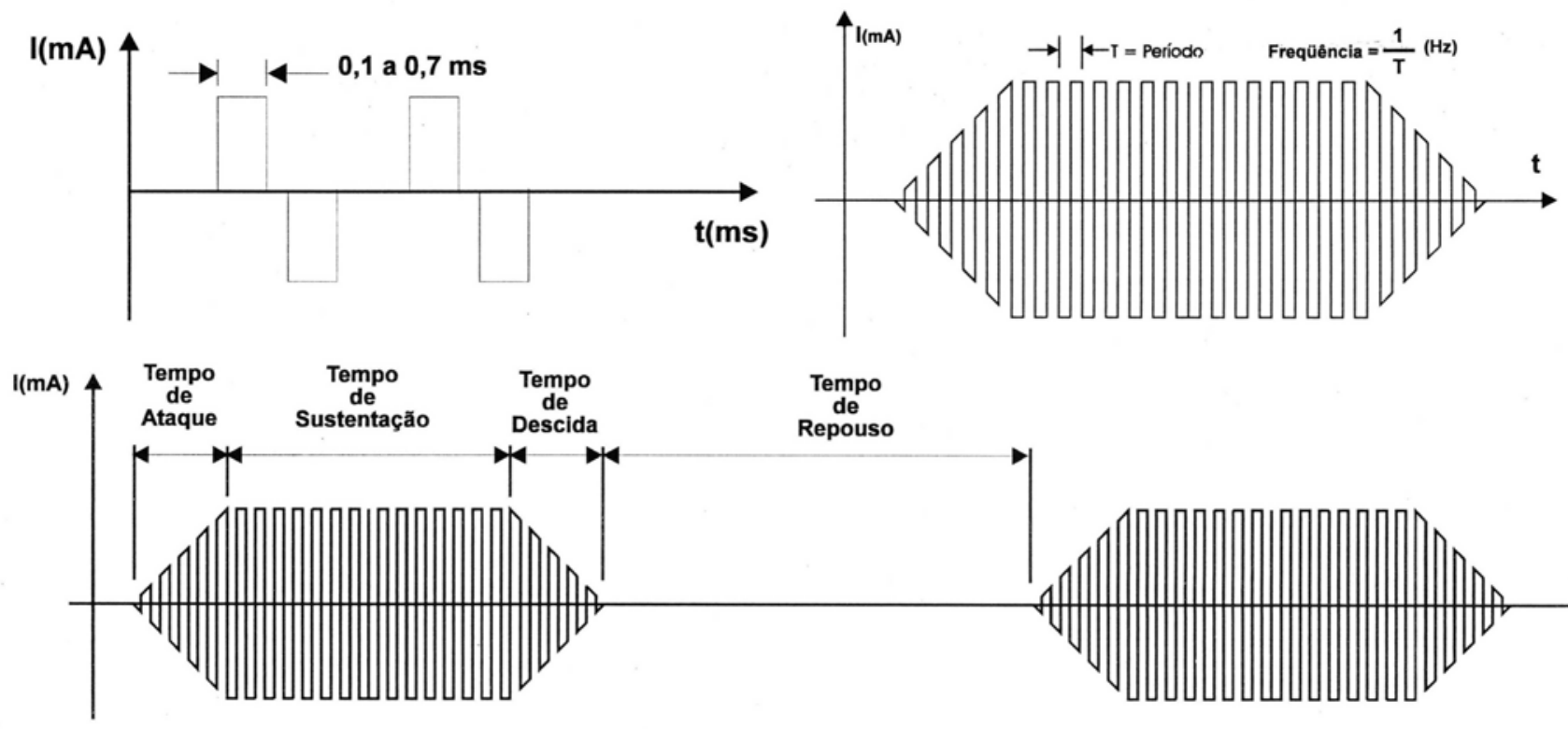


\section{Pacientes com seqüelas de traumatismo crânio-encefálico:}

01 foi estimulado em dorsiflexores do pé;

01 foi estimulado em dorsiflexores do pé e extensores de punho e dedos.

\section{Paciente portador de esclerose múltipla:}

01 foi estimulado bilateralmente no quadríceps.

\section{Resultados}

\section{Pacientes com acidente vascular cerebral:}

14 pacientes apresentaram diminuição de espasticidade com ganho funcional e melhora no padrão de marcha;

12 pacientes apresentaram diminuição do quadro álgico (ombro doloroso);*

07 pacientes não apresentaram alterações;

05 pacientes abandonaram o tratamento.

*O acompanhamento da evolução ocorreu através da informação subjetiva da redução da dor. Não foi realizada uma quantificação do grau de melhora.

\section{Pacientes com traumatismo raquimedular:}

13 pacientes apresentaram diminuição da espasticidade;

02 pacientes não apresentaram alteração.

Resposta dos pacientes com lesão medular ao FES (Avaliação da Espasticidade)

$81,8 \%$ apresentaram diminuição da espasticidade

\section{Pacientes com traumatismo crânio-encefálico:}

02 não apresentaram alteração.

\section{Pacientes com paralisia celebral:}

02 abandonaram o tratamento.

\section{Paciente com esclerose múltipla:}

01 não apresentou alteração.

\section{Resultado da Terapia FES:}

- Abandono:

- Inalterado:

7 pacientes

$12,06 \%$

- Favorável:

12 pacientes

$20,69 \%$

$67,25 \%$

TOTAL:
39 pacientes

58 pacientes

$100 \%$

\section{Conclusões}

Os dados obtidos permitiram comprovar a eficácia da associação das técnicas de estimulação elétrica funcional (FES) e cinesioterapia em pacientes com lesão do neurônio motor superior.

A aplicação regular da estimulação neuromuscular, usando FES, provou ser eficiente no tratamento de pacientes com deficiências motoras causadas por diferentes patologias do sistema nervoso central.

Os movimentos produzidos pela técnica FES são totalmente controláveis e permitem ao paciente uma atitude psicológica positiva, por estar utilizando seus próprios músculos, o que em muito contribui para sua reabilitação física e emocional.

Apesar de ser desejável o conhecimento sobre a técnica FES para que esta torne-se acessível a todos, deve-se observar atentamente as indicações e protocolos técnicos a ela associados. Agindo assim, diminui-se o risco de, no futuro, desacreditar este valioso meio terapêutico que está revolucionando o tratamento das lesões do neurônio motor superior (paralisias e paresias espásticas).

A técnica de FES vem, assim, somar-se ao arsenal de meios terapêuticos já existentes e deverá ser utilizada em conjunto com a cinesioterapia, compondo um programa completo de tratamento, treinamento e reabilitação.

Com este resultado favorável foram ampliados os trabalhos nesta área.

\section{Referências Bibliográficas}

01. GRACANIN, F. - "Aplicação de corrente de baixa freqüência em medicina física e reabilitação, com ênfase especial em estímulo elétrico funcional". RevMed-Reabil. Dez; 20/21: 18-23, 1988.

02. VODOVNIK, L.; BDDJ, T.; KRALJ, A.; GRACANIN, F.; STROJNIK, P. - "Functional electrical stimulation for control of locomotor systems". CRC Critical Review of Bioengineering, Vol. 6, Issue 2, 63-131, Sept. 1981.

03. GRACANIN, F. - "Use of functional stimulation in external control of motor activity and movements of human extremities". Med. Progr. Technol. 4: 149, 1977.

04. GRANAT, M.; KEATING, J.F.; SMITH, A.C.; DELARGY, M.; ANDREWS, B.J. - "The use of functional electrical stimulation to assist gait in patients with incomplete spinal cord injury". Disabil-Rehabil. Apr-Jun; 14(2): 93-7, 1992.

05. GRACANIN, F. - "Use of functional stimulation in rehabilitation of hemiplegic patients". Report 19-P58395-F-012-66; Washington, D.C.: Department of HEW, 1972.

06. GRACANIN, F. - "Estimulação elétrica funcional". Krusen:Tratado de Medicina Física e Reabilitação: 383-397. 
07. BRANDELL, B.R. - " The study and correction of human gait by electrical stimulation". Am-Surg. May; 52(5): 257-63, 1986.

08. MEADQWS, P.M.; MCNEAL, D.R. - "A four-channel IBM PC/AT compatible biphasic pulse generator for nerve stimulation". IEEE-Trans-Biomed-Eng. Jul; 36(7): 802-4, 1989.

09. LEVY, M.; MIZRACHI, J.; SUSAK, Z. - "Recruitment, force and fatigue characteristics of quadriceps muscles of paraplegics isometrically activated by surface functional electrical stimulation". J-Biomed-Eng. Mar; 12(2): 150-6, 1990.

10. STEFANOVSKA, A.; GROS, N.; VODOVNIK, L.; REBERSEK, S.; ACIMOVIC, R. - "Chronic electrical stimulation for the modification of spasticity in hemiplegic patients". Scand-J-Rehabil-Med-Suppl. 17: 115-21, 1988.
11. RAGNARSSON, K.T. - "Functional electrical stimulation and suppression of spasticity following spinal cord injury". Bull-N-Y-AcadMed. Mar-Apr; 68(2): 351-64, 1992.

12. STANIC, U.; ACIMOVIC, R.; GROS, N.: TRNKOCZY, A.; BADJ, T.; KLJAJIK, M. - "Multichannel electrical stimulation for correction of hemiplegic gait". Scand. J. Rehabil. Med. Suppl. 10(2): 75-92, 1978.

13. LIANZA, S. - Estimulação Elétrica Funcional - FES e Reabilitação. Livraria Editora Ateneu. São Paulo, 1993. 